\title{
Stability Analysis of Systems With High VSC Penetration: Where Is the Limit?
}

\begin{tabular}{|c|c|}
\hline Journal: & IEEE Transactions on Power Delivery \\
\hline Manuscript ID & TPWRD-00858-2019.R1 \\
\hline Manuscript Type: & Transactions \\
\hline $\begin{array}{l}\text { Date Submitted by the } \\
\text { Author: }\end{array}$ & 01-Nov-2019 \\
\hline Complete List of Authors: & $\begin{array}{l}\text { Collados-Rodriguez, Carlos; CITCEA-UPC, Electrical Engineering } \\
\text { Cheah-Mane, Marc; CITCEA-UPC, } \\
\text { Prieto-Araujo, Eduardo; CITCEA-UPC, } \\
\text { Gomis-Bellmunt, Oriol; CITCEA-UPC, }\end{array}$ \\
\hline Technical Topic Area : & $\begin{array}{l}\text { FACTS and power electronic applications to ac transmission }< \\
\text { Transmission and Distribution }\end{array}$ \\
\hline Key Words: & $\begin{array}{l}\text { Converters, Frequency control, Power control, Power electronics, } \\
\text { Synchronous generators, Voltage control }\end{array}$ \\
\hline
\end{tabular}

\section{SCHOLARONE ${ }^{m}$ \\ Manuscripts}




\title{
Stability Analysis of Systems With High VSC Penetration: Where Is the Limit?
}

\author{
Carlos Collados-Rodriguez, Marc Cheah-Mane, Member, IEEE, Eduardo Prieto-Araujo, Member, IEEE, and \\ Oriol Gomis-Bellmunt, Senior Member, IEEE
}

\begin{abstract}
The paper addresses the stability of modern voltagesource-converter-dominated power systems, which are experiencing a progressive phase-out of conventional generation. An essential system model is used to capture the fundamental system dynamics, to conduct a thorough mathematical analysis and understand the principles governing network stability in converter-dominated power systems. A detailed analysis is developed to identify the stability limits of this system when voltage source converters operate in grid-following mode. A complete mathematical linear model including the dynamics of the network elements is used to identify these stability limits. Based on this model, a detailed assessment of the influence that the voltage source converter controllers have on the system stability is performed to identify potential stability and reveal the main mechanisms of interaction. Then, a study to identify the minimum synchronous generation to ensure system stability is developed, including analytical expressions of some critical system poles. The mathematical results obtained are validated with a complete non-linear simulation model of the system.
\end{abstract}

Index Terms-Synchronous generator, voltage source converter (VSC), VSC-dominated power systems

\section{INTRODUCTION}

$\mathbf{T}$ HE increasing penetration of renewable energy in the power system is resulting in a high number of power electronics units, in particular Voltage Source Converters (VSCs), connected to the grid [1]. Such increase of renewable generation will motivate a progressive phase-out of synchronousgenerator-based power plants, leading to a reduction of the short-circuit current and inertia of the system [1]. Additional power electronics units for transmission and distribution applications, such as HVDC links, FACTS or energy storage, are also contributing to increase the penetration of VSC-based elements.

Power system dynamics will be increasingly dominated by VSC controllers, posing new challenges on the system stability [1]. Thus, in a VSC-dominated power system, a new breed of interactions might appear between different network elements, requiring further studies and new methodologies to assess the system stability. While these issues are going to appear in most modern power systems, small systems (such as geographical islands) are expected to be the first to experience these stability challenges.

Part of the aforementioned issues have been addressed in the fields of weak grids and low inertia systems. In terms of weak grids, several studies have addressed the impact of the PLL [2], [3], interactions between outer control loops of the converter [4], [5] and possible resonances between the converter control and the grid impedance [4], [6]. Several VSC control strategies have been proposed to enhance the converter response in such conditions, e.g. power synchronization control [7] or advanced vector control [5]. Other articles illustrate the importance of providing frequency support from converters when connected to a low-inertia system [8], [9].

Currently, operation of VSCs connected to the grid is mainly based on grid-following controls. Such operation might compromise the system stability for a high penetration of power-electronics units. As an alternative, grid-forming controls have been suggested to improve the system stability and enable a $100 \%$ penetration of converter-based elements. A number of control approaches for grid-forming have been presented in the literature [10]-[14]. However, the penetration limits of VSCs operating with grid-following control must be identified before the transition to grid-forming operation. Therefore, the interactions between the grid-following VSCs and Synchronous Generators (SGs) must be well understood as well as the minimum SG share to ensure system stability.

The stability of power systems including both VSCs and SGs has been analyzed in [15]-[19]. Potential interactions between VSC voltage control and the SG exciter are analyzed in [15] through an eigenvalue analysis while the size of the VSC is progressively increased. Also, the relationship between reactive power and rotor angle stability with high penetration of wind generation is studied in [16]. Grid-following controls with frequency droop control and virtual inertia are analyzed in [17] for different scenarios replacing SGs by VSCs. Moreover, in [18], [19] a sensitivity analysis of the stability is presented depending on the converter penetration, line length and SG model simplifications. However, a full sensitivity analysis of the VSC control parameters for different grid-following operational modes has not been detailed in the literature.

This paper presents a stability analysis of systems with high VSC penetration, considering grid-following operation and a reduction of the SG share. While the previous references provide interesting results on the effect of the SG share in a system dominated by power converters, this article provides a deeper insightful analysis to understand the limits of the gridfollowing operation. In particular, the following contributions can be summarized as follows:

- Study the impact of different grid-following VSC controllers (active power control, voltage control, frequency control) on the system stability.

- Detailed analysis on the impact that a progressive SG reduction has on the stability of VSC-dominated power systems, considering grid-following VSC controllers.

- Derivation of a comprehensive analysis to assess stability and reveal the main mechanisms of interaction, based on an essential system model.

- Interaction assessment considering the combination of an eigenvalue analysis (including a color group classification) and a participation factor interaction matrix. 
- Analytical derivation of some of the critical system poles when SG is reduced. A simplified expression is obtained to calculate the stability limit in such conditions.

The article is structured as follows. In Section II, the essential model to develop the detailed stability analysis is derived (linear and complete non-linear models). This model is used in Section III to assess the impact of VSC controllers in network stability and in Section IV to obtain the minimum synchronous generation required. Section $\mathrm{V}$ discusses the main findings of the article.

\section{SYSTEM DESCRIPTION AND MODELING}

The studied system is based on a small AC grid with SGs and a high penetration of VSCs, i.e. VSC-HVDC links, wind and photovoltaic power plants, as shown in Fig. 1. Specifically, the essential model that represents the fundamental dynamics of such system consists on an SG, which is an aggregated representation of all the thermal-based generation, a VSC, which aggregates all the VSC-based elements and an aggregated power demand. Several publications have proposed methods to study aggregated models of SGs [20]-[22] and VSCs [23][26], which are mostly based on identifying the coherence among the different units. This topic is beyond the scope of this paper and the SG and VSC parameters are based on single units.

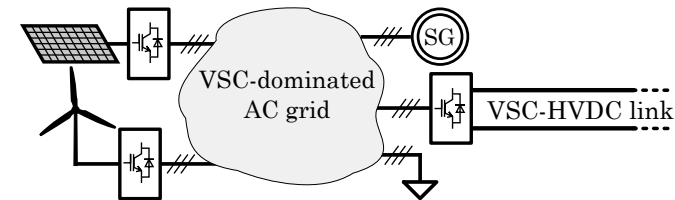

(a) General scheme

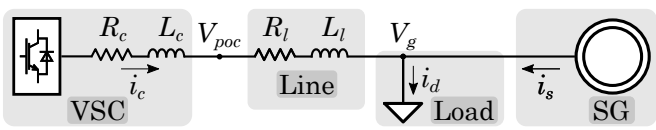

(b) Essential model

Fig. 1. Studied system with high VSC penetration

Two different models have been implemented with the previous components: a complete non-linear model to perform time domain simulations and a linear model to develop the mathematical analysis. The model is divided in three subsystems, SG, VSC and AC grid, which are detailed in the following subsections. Also, the complete linear model is described.

\section{A. Synchronous generator}

The SG representation contains a generator model, an excitation system, steam turbine dynamics and a governor, as shown in Fig. 2. A power system stabilizer is not considered in the SG model since its control design is highly dependent on the oscillations of particular system.

1) Generator: the electrical circuit of the $\mathrm{SG}$ is represented as a round rotor, including six winding circuits, which are expressed in the SG Reference Frame (SG-RF) [27]: the $q$ and $d$ stator windings, the field winding in the $d$-axis and two $q$ and one $d$ damper windings. Also, the SG mechanical dynamics are represented as single-mass model, with an inertia constant $J$.

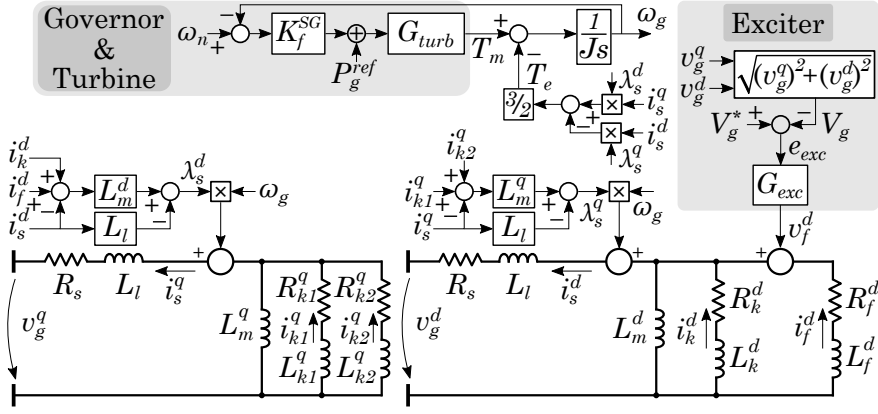

Fig. 2. Equivalent representation of the $\mathrm{SG}$

2) Exciter: the exciter is based on the IEEE model AC4 [28], which is represented as:

$$
G_{e x c}(s)=\frac{T_{C} s+1}{T_{B} s+1} \frac{K_{A}}{T_{A} s+1} K_{f s}
$$

where $T_{B}$ and $T_{C}$ are the lag and lead time constants and $K_{A}$ and $T_{A}$ are the gain and time constant of the regulator. The constant $K_{f s}$ is used to represent field magnitudes in the stator circuit and is expressed as $K_{f s}=\left(R_{f}^{d} / L_{m}^{d}\right) V_{g}$.

3) Governor and turbine: the governor includes a frequency regulator based on conventional frequency-power (f-P) droop control, as shown in Fig. 2. The steam turbine includes a high pressure turbine, a reheater and a low pressure turbine [29] and is modeled as:

$$
G_{\text {turb }}(s)=\left(K_{H P}+\frac{K_{L P}}{T_{R H} s+1}\right) \frac{1}{\omega_{g}}
$$

where $K_{H P}$ and $K_{L P}$ are the fractions of the mechanical torque applied by the high and low pressure turbines respectively and $T_{R H}$ is the time constant of the reheater.

\section{B. VSC control}

The VSC model contains a conventional vector control [30], including a Phase-Locked Loop (PLL), an inner current control loop, active power and $\mathrm{AC}$ voltage outer control loops and a frequency control, as shown in Fig. 3. The converter control is implemented in the VSC Reference Frame (VSC-RF) using $q d$ variables.

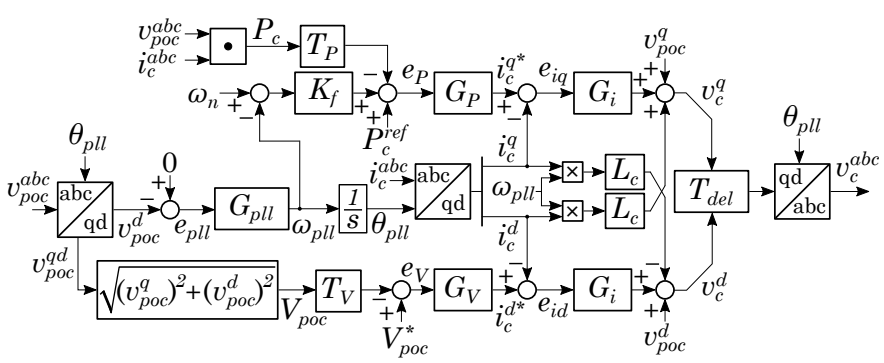

Fig. 3. VSC control scheme

1) Phase-locked loop: the PLL is required to track the network voltages and ensure an adequate control of the VSC. This PLL aligns the VSC-RF to the $q$-axis of the SG-RF with a PI controller $G_{p l l}(s)$. The output of this controller is the estimated grid frequency, $\omega_{p l l}$, which is integrated to obtain 
the angle of the VSC-RF, $\theta_{p l l}$. In the non-linear model, $\theta_{p l l}$ is used to obtain $q d$ variables applying the Park transformation. The transfer function of the PLL controller is:

$$
G_{p l l}(s)=k_{p-p l l}+\frac{k_{i-p l l}}{s}
$$

where $k_{p-p l l}$ and $k_{i-p l l}$ are the proportional and integral gains of the controller, which can be calculated based on the AC voltage amplitude and the bandwidth of the PLL [31].

2) Current control loop: the inner current control loop is implemented using two PI controllers, $G_{i}(s)$, and decoupling terms, $\omega_{p l l} L_{c}$, as shown in Fig. 3. The PI controllers are designed based on the Internal Model Control (IMC) method [30], which defines a first-order response with a time constant $\tau_{i}$. This time constant is normally set in the few milliseconds range. Then, the PI controllers are expressed as:

$$
G_{i}(s)=k_{p-i}+\frac{k_{i-i}}{s}, \quad k_{p-i}=\frac{L_{c}}{\tau_{i}}, \quad k_{i-i}=\frac{R_{c}}{\tau_{i}}
$$

The delay of the voltage modulation is introduced as a firstorder function, $T_{d e l}$, with a time constant $\tau_{m}$.

3) AC power and voltage control: these loops are based on PI controllers, $G_{P}(s)$ and $G_{V}(s)$, respectively, as shown in Fig. 3. The controller outputs correspond to the current references of the inner control loop. Also, two first-order filters, with time constants $\tau_{P}$ and $\tau_{V}$, have been added to the power and voltage measurements, represented as $T_{P}$ and $T_{V}$ in Fig. 3.

4) Frequency control: the frequency support is implemented as a conventional f-P droop control. The droop characteristic, $R$, and gain, $K_{f}$, are defined as [29]:

$$
R(\%)=\frac{\Delta \omega(\%)}{\Delta P_{c}(\%)} 100 ; \quad K_{f}=\frac{S_{V S C}}{w_{n} R}
$$

where $\Delta \omega$ is the frequency variation associated to the power variation $\Delta P_{c}, S_{V S C}$ is the VSC rated power and $\omega_{n}$ is the nominal frequency. Then, the reference power is:

$$
P_{c}^{*}=K_{f}\left(w_{n}-w_{p l l}\right)+P_{c}^{r e f}
$$

where $P_{c}^{r e f}$ is the reference power at $\omega_{n}$.

\section{AC grid modeling}

The AC grid model includes all the system impedances and a load. The converter filter is modeled as an RL circuit, as shown in Fig. 1b. The AC line interconnecting the SG and the converter is also modeled as an RL equivalent, which is sufficient to represent a short distance line in a small system. The load is represented as an equivalent resistance, which represents a power demand at the nominal AC voltage.

\section{Complete linear system}

The complete linear system is represented in two rotating reference frames, such that the AC grid and the $\mathrm{SG}$ are expressed in the SG-RF while the VSC control is expressed in the VSC-RF. The variables in the SG-RF, $x^{q d}$, can be expressed in the VSC-RF using the following rotation:

$$
\Delta x^{q d c}=\mathbf{T}_{\mathbf{c}}\left(\Delta x^{q}, \Delta x^{d}, \Delta e_{\theta}\right)^{T}
$$

$$
\mathbf{T}_{\mathbf{c}}=\left(\begin{array}{ccc}
\cos \left(e_{\theta_{0}}\right) & -\sin \left(e_{\theta_{0}}\right) & -\sin \left(e_{\theta_{0}}\right) x_{0}^{q}-\cos \left(e_{\theta_{0}}\right) x_{0}^{d} \\
\sin \left(e_{\theta_{0}}\right) & \cos \left(e_{\theta_{0}}\right) & \cos \left(e_{\theta_{0}}\right) x_{0}^{q}-\sin \left(e_{\theta_{0}}\right) x_{0}^{d}
\end{array}\right)
$$

where $x^{q d c}$ are the variables in the VSC-RF, $T_{c}$ is the linearized rotation matrix, $e_{\theta}$ is the angle difference between the SG-RF and the VSC-RF. The variables with subindex 0 refer to the initial value at the linearization point. Analogously, an anti-rotation matrix is used to express $x^{q d c}$ in the SG-RF:

$$
\begin{gathered}
\Delta x^{q d}=\mathbf{T}_{\mathbf{c}}^{-\mathbf{1}}\left(\Delta x^{q c}, \Delta x^{d c}, \Delta e_{\theta}\right)^{T} \\
\mathbf{T}_{\mathbf{c}}^{-\mathbf{1}}=\left(\begin{array}{ccc}
\cos \left(e_{\theta_{0}}\right) & \sin \left(e_{\theta_{0}}\right) & -\sin \left(e_{\theta_{0}}\right) x_{0}^{q c}+\cos \left(e_{\theta_{0}}\right) x_{0}^{d c} \\
-\sin \left(e_{\theta_{0}}\right) & \cos \left(e_{\theta_{0}}\right) & -\cos \left(e_{\theta_{0}}\right) x_{0}^{q c}-\sin \left(e_{\theta_{0}}\right) x_{0}^{d c}
\end{array}\right)
\end{gathered}
$$

The angle difference between both RFs is obtained as:

$$
\Delta e_{\theta}(s)=\left(\Delta \omega_{p l l}(s)-\Delta \omega_{g}(s)\right) / s
$$

The PLL can introduce deviations in the estimated grid frequency $\omega_{\text {pll }}$, specially during voltage transients, which results in an angle disturbance between both RFs in (11).

Combining all the previous subsystems, a complete linear model of the system is obtained, as shown in Fig. 4. This linear model is expressed as:

$$
\begin{aligned}
& \Delta \dot{x}=\mathbf{A} \Delta x+\mathbf{B} \Delta u \\
& \Delta y=\mathbf{C} \Delta x+\mathbf{D} \Delta u
\end{aligned}
$$

where $\Delta x, \Delta u$ and $\Delta y$ are the variations of state, input and output vectors. In particular, the state vector $x$ is defined as:

$$
\begin{aligned}
& \text { VSC }
\end{aligned}
$$

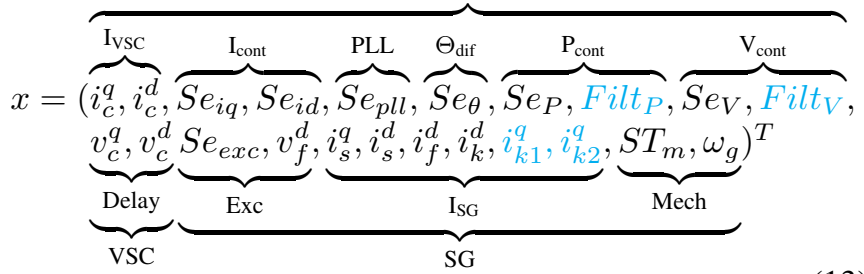

which represents the variables of the SG and VSC from Figs. 2 and 3. It should be noted that $e$ refers to the error between a variable and its reference value and $S$ refers to the integral of a variable. The input vector $u$ is defined as:

$$
u=\left(R_{d}, P_{c}^{r e f}, V_{\text {poc }}^{*}, P_{g}^{r e f}, V_{g}^{*}\right)^{T}
$$

where $R_{d}$ is the equivalent load resistance, $P_{c}^{r e f}$ and $V_{\text {poc }}^{*}$ are inputs of the VSC control and $P_{g}^{r e f}$ and $V_{g}^{*}$ are inputs of the SG control. The output vector $y$ can be chosen arbitrarily depending on the variables to observe.

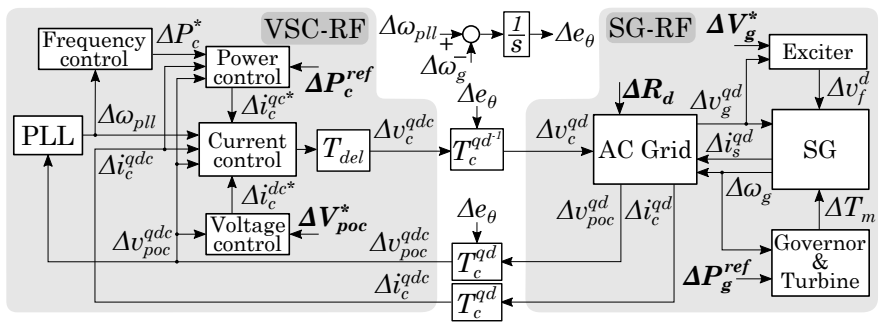

Fig. 4. Linear model of the system with SG and VSC

The linear model in Fig. 4 has been validated with the complete non-linear model. Fig. 5 shows the response of both models after a load variation of $1 \%$, showing the adequacy of the linear model. 

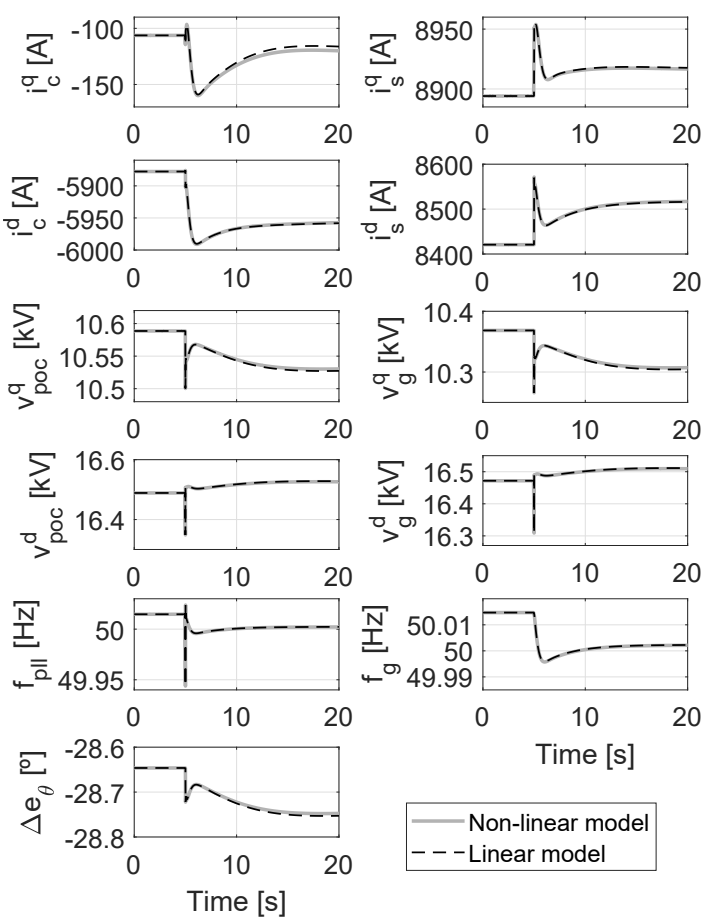

Fig. 5. Simulation results of the non-linear and linear models

\section{IMPACT OF VSC CONTROL}

In this section, potential interactions between the VSC and SG are identified due to modifications of VSC control parameters. The study is based on an eigenvalue analysis from the small-signal model of the system, providing information about the poles, i.e. frequency and damping of the modes, and the participation factors (PFs) of the system [32]. A comprehensive analysis of the potential system interactions is developed based on the following factors:

- Participating variables: coupling between SG and VSC, i.e. interaction, is considered when the PFs associated to variables from both elements are high enough.

- Damping: low-damped modes are related to critical poles. Negative-damped modes, i.e. poles with positive real part, show system instabilities.

- Pole sensitivity: the location of a pole can be sensitive to the modification of control parameters even if the PFs of those control variables are low.

The poles have been represented with different colors according to the variables with the highest PFs, as shown in Fig. 6a. The PFs have been normalized between 0 (no participation) and 1 (maximum participation) for each mode. Then, it is assumed that variables have a significant effect on a mode when their PFs are greater than 0.3. Also, the PFs are represented in a matrix format where the variables are grouped as shown in (13). Each component of the matrix represents the PF values for several control parameter variations (to be read from left to right inside the cell). The PF values are expressed using a grayscale color code, ranging from white (no participation) to black (maximum participation). This is shown in Fig. 6b, where $n$ conditions correspond to an increase of a control parameter $k_{\text {cont }}$ from $k_{\min }$ to $k_{\max }$.

\begin{tabular}{l|l}
\multicolumn{1}{c|}{ Simple colors } & Combined colors \\
\hline - VSC Currents & VSC Currents + VSC Control \\
VSC Control & SG Currents + VSC Currents \\
- SG Currents & SG Currents + VSC Control \\
SG Exciter & SG Currents + VSC Currents + VSC Control \\
- SG Mechanics & SG Currents + SG Exciter \\
& SG Exciter + VSC Control \\
& SG Currents + SG Exciter + VSC Control \\
& SG Currents + SG Frequency \\
& SG Currents + SG Frequency + VSC Control
\end{tabular}

(a) Pole colors depending on variables with high participation

\begin{tabular}{|c|c|c|c|c|c|c|}
\hline Group & Var & Pole1 & Pole2 & & $k_{\text {cont }} \quad k_{\max }$ & PF value \\
\hline \multirow{2}{*}{ G1 } & Var1 & & & & & $\begin{array}{l}\text { High } \\
\text { Medium }\end{array}$ \\
\hline & Var2 & & & & $\begin{array}{c}\cdots n-1 \\
\text { Cases }\end{array}$ & Low \\
\hline
\end{tabular}

(b) PF matrix representation according to variation of $k_{\text {cont }}$

Fig. 6. Description of the results

Fig. 7 shows four case studies, implementing different VSC outer loops explained in Section II. Case 1 represents the VSC with a basic control scheme, including the PLL, inner current control and outer active power control. Voltage and frequency control loops are gradually introduced in Cases 24. The impact of the control loops is analyzed by modifying the controller parameters. In particular, the parameters of the control loops marked in gray in Fig. 7 are modified for each case study. All case studies are based on the parameters in the Appendix.

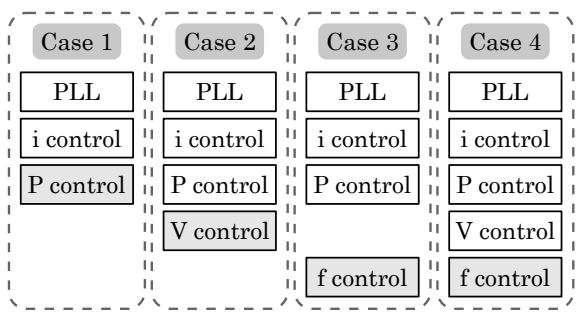

Fig. 7. Control loops implemented in the case studies

A. Case 1. Power control

The effect of modifying parameters of the active power control is analyzed in Case 1. The PI control parameters are scaled using the variable $\alpha_{P}$, which is increased from 0.1 to 1 with variations of 0.1 . Fig. 8a shows the pole diagram and Fig. $8 \mathrm{~b}$ shows the PF matrix following the variation of $\alpha_{P}$. The modification of power control parameters only affects the mode associated to active power. This is observed from pole 14 , which has a high participation of the group $P_{\text {cont }}$ in Fig. 8b. The remaining poles are not sensitive to the power control variation. Also, three potential interactions between the generator and the converter are identified. This is observed from poles 5 and 6 , which show a high participation of groups $I_{S G}, I_{V S C}$ and Delay, poles 7 and 8, with high participation of groups $P L L$ and $I_{S G}$, and pole 14, with high participation of groups $P_{\text {cont }}$ and $I_{S G}$.

Table I summarizes the pole information shown in Fig. 8, which is considered as a reference case. Most of the poles represent non-oscillatory modes or low frequency oscillations, while only three pair of poles represent supersynchronous modes, i.e. frequencies above $50 \mathrm{~Hz}$. Modes with the lowest damping are related to SG mechanics (poles 18 and 19) and PLL (poles 7 and 8). However, these modes do not cause significant oscillations due to the high damping level $(>0.48)$. 
1

2

3

4

5

6

7

8

9

10

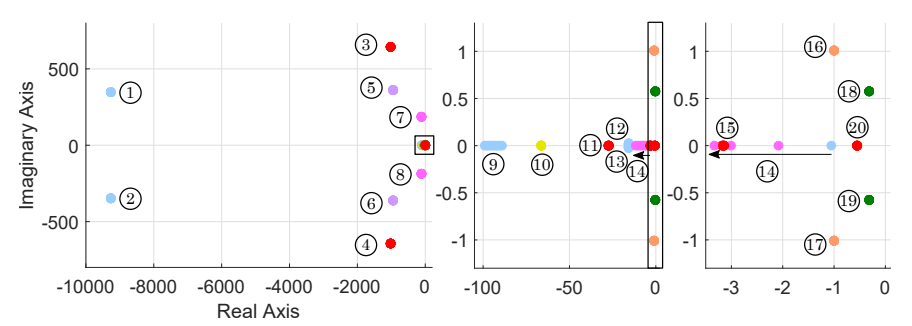

(a)

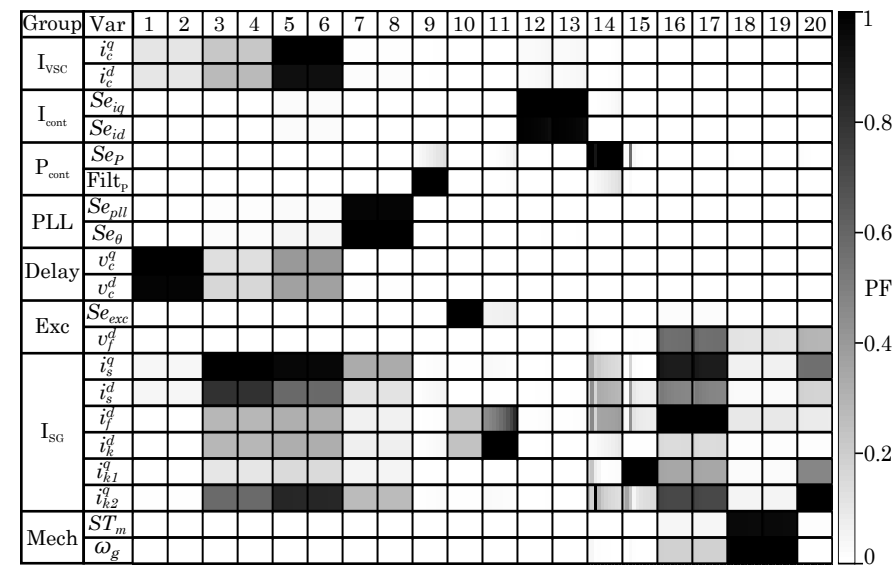

(b)

Fig. 8. Results of Case 1: (a) Pole diagram; (b) PF matrix

TABLE I

Pole CHARACTERISTICS IN CASE 1

\begin{tabular}{|c|c|c|c|c|}
\hline Pole & Group/s & Oscil. Freq. & Damping ratio & Interaction \\
\hline 1,2 & Delay & $55 \mathrm{~Hz}$ & $>0.99$ & - \\
\hline 3,4 & $\mathrm{I}_{\mathrm{SG}}$ & $102 \mathrm{~Hz}$ & 0.846 & - \\
\hline 5,6 & $\mathrm{I}_{\mathrm{VSC}}, \mathrm{I}_{\mathrm{SG}}$ & $58 \mathrm{~Hz}$ & 0.934 & Yes \\
\hline 7,8 & PLL, , ISG & $30 \mathrm{~Hz}$ & 0.518 & Yes \\
\hline 9 & Filt & $0 \mathrm{~Hz}$ & 1 & - \\
\hline 10 & Exc & $0 \mathrm{~Hz}$ & 1 & - \\
\hline 11 & $\mathrm{I}_{\mathrm{SG}}$ & $0 \mathrm{~Hz}$ & 1 & - \\
\hline 12,13 & $\mathrm{I}_{\text {cont }}$ & $0 \mathrm{~Hz}$ & 1 & - \\
\hline 14 & $P_{\text {cont }}, I_{S G}$ & $0 \mathrm{~Hz}$ & 1 & Yes \\
\hline 15 & $\mathrm{I}_{\mathrm{SG}}$ & $0 \mathrm{~Hz}$ & 1 & - \\
\hline 16,17 & $\mathrm{Exc}, \mathrm{I}_{\mathrm{SG}}$ & $0.16 \mathrm{~Hz}$ & 0.704 & - \\
\hline 18,19 & Mech & $0.09 \mathrm{~Hz}$ & 0.481 & - \\
\hline 20 & $\mathrm{I}_{\mathrm{SG}}$ & $0 \mathrm{~Hz}$ & 1 & - \\
\hline
\end{tabular}

\section{B. Case 2. Power and voltage control}

In this case, the AC voltage control is added to the VSC. The PI control parameters are scaled using a variable $\alpha_{V}$, which is increased from 0 to 1 with variations of 0.1 . The VSC voltage control introduces two new poles in the system, poles 21 and 22 in Fig. 9a. An interaction between the VSC voltage control and the exciter is identified, which corresponds to an oscillation mode with frequency in the range of $0.01 \mathrm{~Hz}$ and high damping $(>0.9)$. This interaction is represented by poles 20 and 22, which becomes a complex conjugate pair while $\alpha_{V}$ is increased. Also, the PF matrix in Fig. 9b shows that these poles have a high participation of groups $V_{\text {cont }}$ and Exc.

This interaction could be understood from the reactive power exchange between the VSC and the SG. The VSC voltage control provides the reactive power needed to regulate the POC voltage at the desired level, e.g. $1 \mathrm{pu}$ in this case. Excluding the reactive power consumption of the line, the remaining reactive power provided by the converter is compensated by the SG, causing the interaction between both elements. In a more complex and meshed grid, this interaction may be avoided as the reactive power provided by a VSC is not compensated by a single SG and could be shared by different elements of the system.

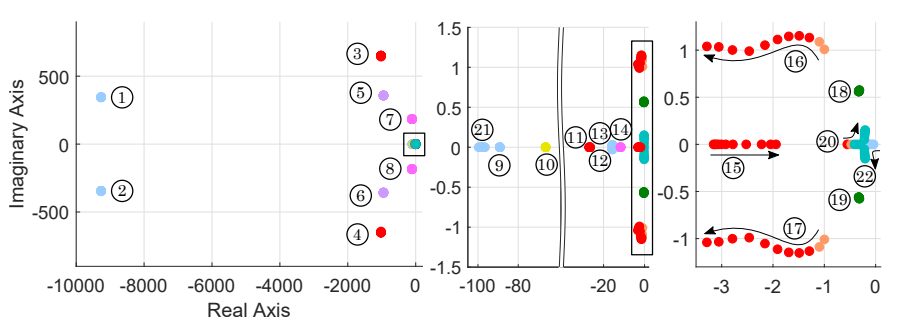

(a)

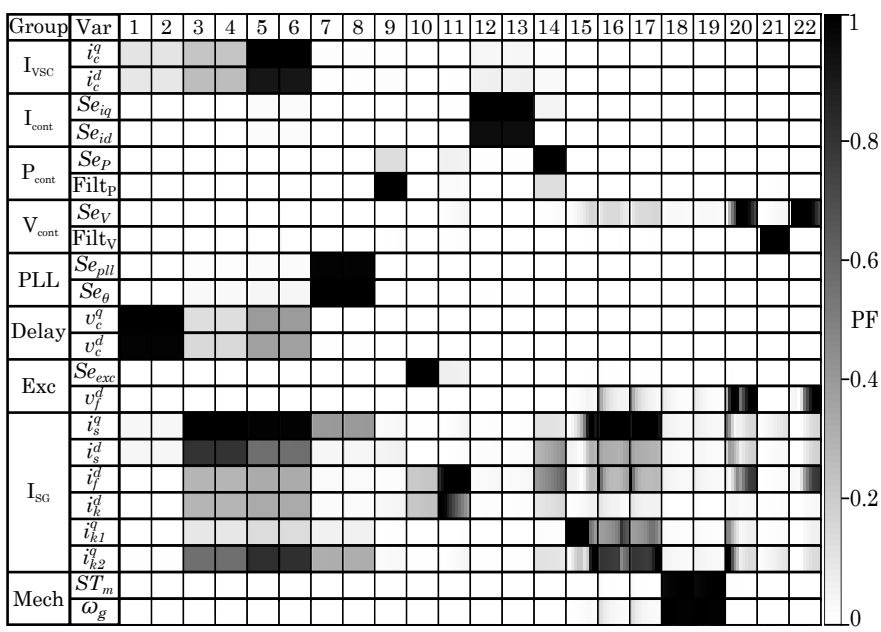

(b)

Fig. 9. Results of Case 2: (a) Pole diagram; (b) PF matrix

C. Case 3. Power and frequency control

The impact of the VSC frequency control is analyzed in Case 3. The f-P droop characteristic of the converter $R$ is modified, considering the following values: $100 \%, 50 \%, 25 \%$, $10 \%, 5 \%, 4 \% 3 \%, 2.5 \%, 2 \%, 1.5 \%$ and $1 \%$. Below 5\%, more $R$ values are tested since the pole sensitivity is higher, as shown in Fig. 10a. When the VSC frequency control is introduced, two main effects have been identified:

- The grid frequency is coupled with control or electrical variables instead of the mechanical torque of the turbine.

- PLL and power control of the VSC interact with the SG.

Initially, the grid frequency $\omega_{g}$ is coupled with the mechanical torque of the SG turbine, as in Case 1. Then, when the contribution of the VSC frequency control is increased, i.e. $R$ is reduced, $\omega_{g}$ is coupled with the SG currents, which results in a faster $\omega_{g}$ response. This is observed from poles 14 and 18 in Fig. 10b. Poles 18 and 19 are related to the group Mech for $R$ values higher than $10 \%$, i.e. low contribution of the VSC frequency control. Then, when $R$ is reduced, pole 18 is coupled with other poles (first with pole 20 and later with pole $15)$, becoming complex conjugate poles, which results in an oscillatory mode with high participation of $\omega_{g}$ and the group $I_{S G}$. This oscillation mode presents similar frequency as the 


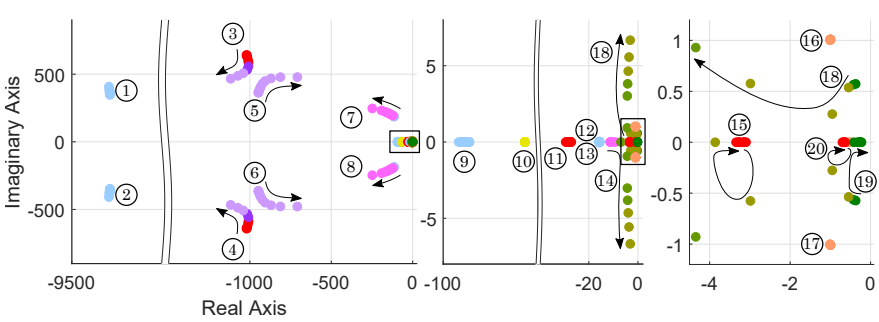

(a)

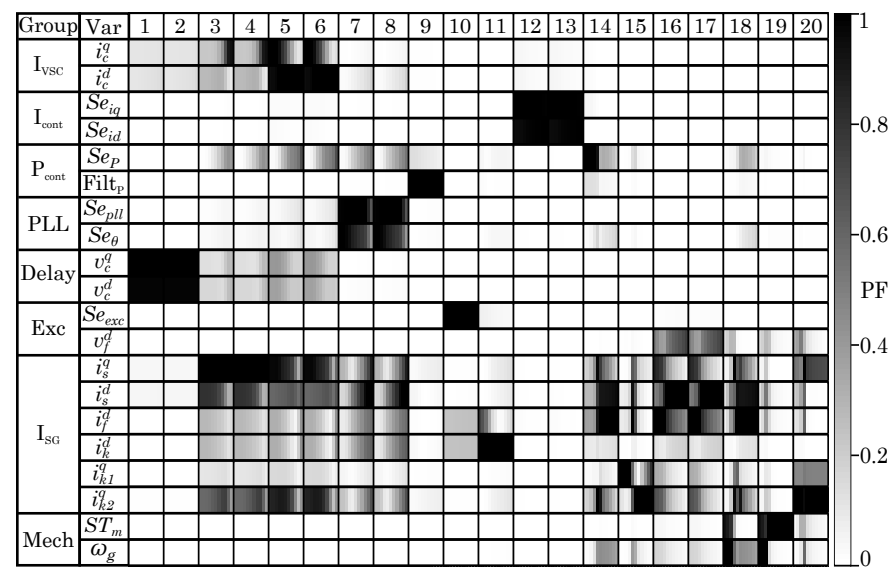

(b)

Fig. 10. Results of Case 3: (a) Pole diagram; (b) PF matrix

mechanical poles in Case 1. Finally, when $R$ is even lower, pole 18 becomes complex conjugate with pole 14, which was related to groups $P_{\text {cont }}$ and $I_{S G}$. As a result, $\omega_{g}$ is related to an oscillation mode with higher frequency (around $1 \mathrm{~Hz}$ ) and high participation of electrical and control variables of groups $P_{\text {cont }}$ and $I_{S G}$.

This interaction reveals the relationship between the active powers of the SG and the VSC and grid frequency. A VSC with a high contribution to frequency support will compensate any load variation, avoiding grid frequency deviations. This leads to a faster response of the grid frequency, as it depends on the VSC power instead of the mechanical torque of the machine.

In addition, the interaction between PLL and power control of the VSC and the SG is also affected due to the introduction of the VSC frequency control. This can be observed in poles 7 and 8 in Fig. 10b, which present a high participation of groups $P L L, P_{\text {cont }}$ and $I_{S G}$. The frequency of this oscillating mode is around $30 \mathrm{~Hz}$ with a damping greater than 0.5 .

This interaction is caused by a closed loop in the VSC control, which contains the PLL, the frequency control and the power control. The PLL is aligned to the $d$-axis of the grid voltage, providing the estimated frequency $\omega_{p l l}$ which is used in the frequency control. As a result, a variation of the grid voltage may lead to an $\omega_{\text {pll }}$ deviation, causing a disturbance in the converter power that can be extended to the rest of the system.

Another interaction arises between the VSC and SG currents, observed from poles 3 and 4 . However, this interaction is not significant since the associated damping increases as the contribution of the VSC frequency control is higher.

\section{Case 4. Power and voltage control and frequency support}

Fig. 11 shows the results obtained when all the control loops are included. In this case, only the frequency control is modified, since the poles are more sensitive to the parameters of this control loop. Then, $R$ is modified as in Case 3 and $\alpha_{V}$ is equal to 1 . This scenario combines the effects observed in Case 2 and 3:

- The VSC voltage control interacts with the exciter (poles 20 and 22).

- PLL and power control of the VSC are coupled with SG currents (poles 7 and 8).

- The grid frequency is coupled with the SG currents (poles 16 and 17).

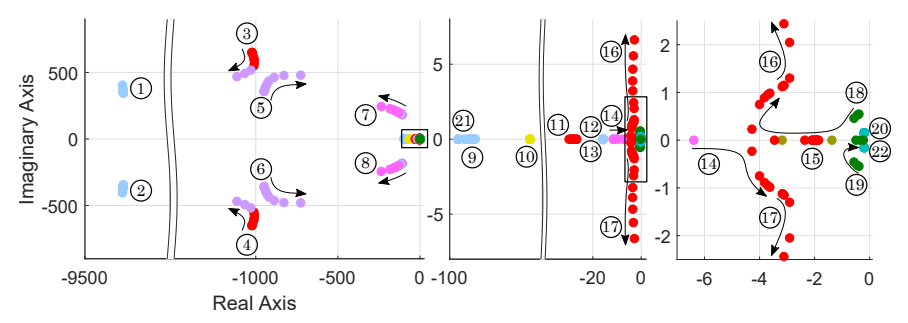

(a)

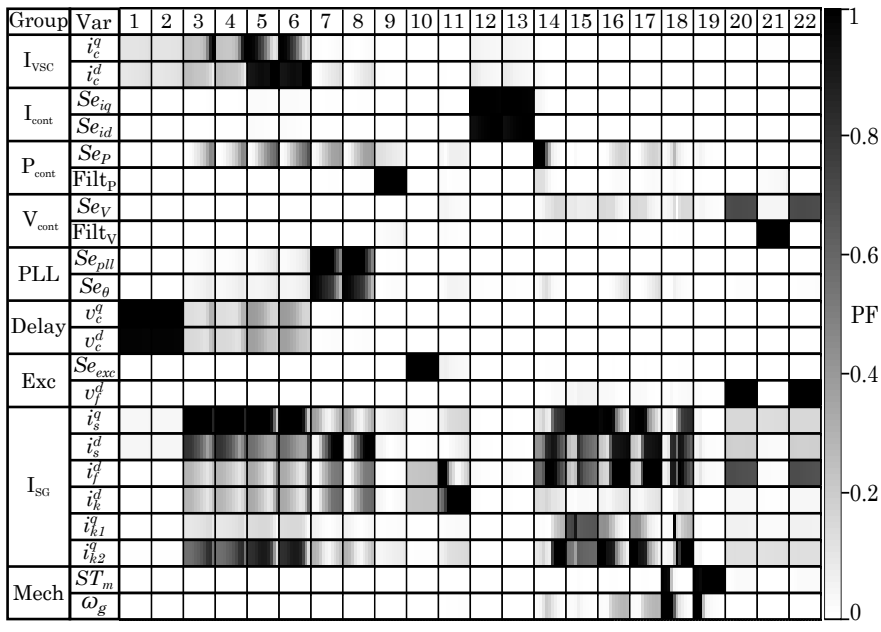

(b)

Fig. 11. Results of Case 4: (a) Pole diagram; (b) PF matrix

\section{Minimum Synchronous Generation}

In this section, the minimum synchronous generation required to ensure stability is identified. The SG share is reduced gradually until the system becomes unstable. Such SG share is defined as the ratio between the SG rated power $S_{S G}$ and the total rated power generation in the system $S_{T}=S_{S G}+S_{V S C}$. Initially, $S_{S G}$ is $500 \mathrm{MVA}$, which corresponds to a SG share of $50 \%$, based on the data in the Appendix. The minimum $S_{S G}$ is evaluated for the case studies in Fig. 7. In all cases, it is assumed the SG active power is maintained at $70 \%$ of $S_{S G}$. Also, analytical expressions of the critical poles are studied.

\section{A. Case 1. Power control}

The rated power of the SG is reduced from 500 MVA to $50 \mathrm{MVA}$ with variations of $50 \mathrm{MVA}$. Also, two more values of $S_{S G}$ are tested: $25 \mathrm{MVA}$ and $10 \mathrm{MVA}$, which correspond to SG shares of $5 \%$ and $2 \%$ respectively. The system remains 
stable even for low SG shares, as shown in Fig. 12. Reducing the SG power mainly modifies its internal impedances, leading to large changes in the dynamics of the SG currents. This is observed from poles related to the SG currents, specially poles 3 and 4, which become very low damped, but none of them is positive.

A non-linear simulation has been performed in order to validate these results. The rated power of the SG has been set to $25 \mathrm{MVA}$, i.e. an SG share of $5 \%$. The system remains stable after a load change of $1 \%$ at $5 \mathrm{~s}$, as shown in Fig. 13. As the VSC does not provide any support, all the power demand increase has to be compensated by the SG, leading to a high frequency reduction. Then, despite the small-signal analysis does not find any instability, the system cannot operate with such a high VSC penetration in PQ mode since any variation in the power demand could cause an excessive frequency reduction.

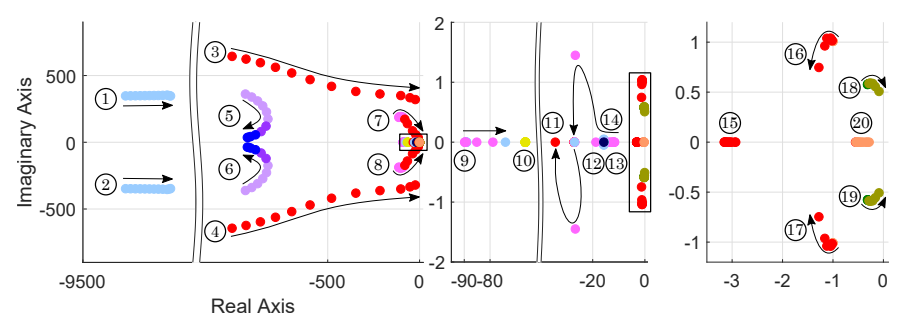

Fig. 12. Pole diagram of Case 1 when $\mathrm{S}_{\mathrm{SG}}$ is reduced

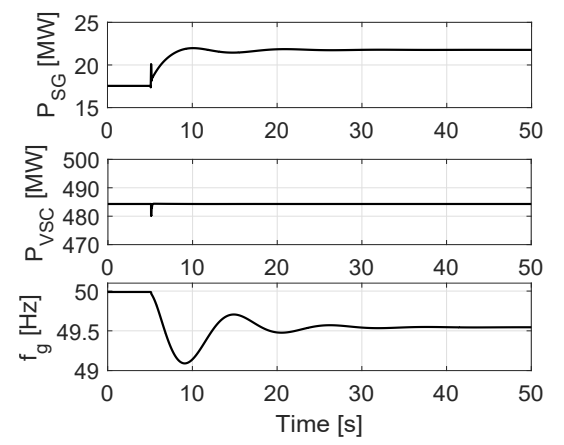

Fig. 13. Time domain results of Case 1 with a SG share of $5 \%$

\section{B. Case 2. Power and voltage control}

In this case, $S_{S G}$ is reduced from 500 MVA to 50 MVA with variations of $50 \mathrm{MVA}$. The stability limit is identified between 35 MVA and 30 MVA (6.5\% and 5.7\% of SG share). Such limit corresponds to the interaction between the VSC voltage control and the exciter identified in Section III-B (poles 20 and 22 in Fig. 14), which leads to a non-oscillatory angle instability [29].

A reduction of the $\mathrm{SG}$ share implies an increase in the VSC active power injection, which causes a larger voltage drop through the line that connects the converter to the SG and load. Since the VSC regulates the POC voltage, reactive power from the converter must be increased. Then, as mentioned in Section III-B, the reactive power compensated by the SG also increases. A high reactive power exchange in the SG leads to an increase of the internal angle of the generator $\delta_{S G}$, which results in a reduction of the SG synchronising torque. An angle instability is produced when the synchronising torque is not enough to keep the machine in synchronism. This relation between the reactive power and angle stability has been reported in the literature [33]. A similar interaction was analyzed in [16], where the effect that full-converter wind turbines have on the SG angle stability was studied.

The instability is validated with non-linear time domain simulations, presented in Fig. 15. A small variation of $V_{p o c}$ reference at the VSC from $0.995 \mathrm{pu}$ to $1 \mathrm{pu}$ at $10 \mathrm{~s}$ increases the SG angle until loss of synchronism when the SG power is 30 MVA. This agrees with the positive real pole 20 in Fig. 14. It is observed that the system remains stable for a higher SG power, e.g. 35 MVA. The stability range can be extended with a proper operation of the converter. For example, keeping the $V_{\text {poc }}$ reference to $0.995 \mathrm{pu}$, the system is stable for 30 MVA, also shown in Fig. 15.

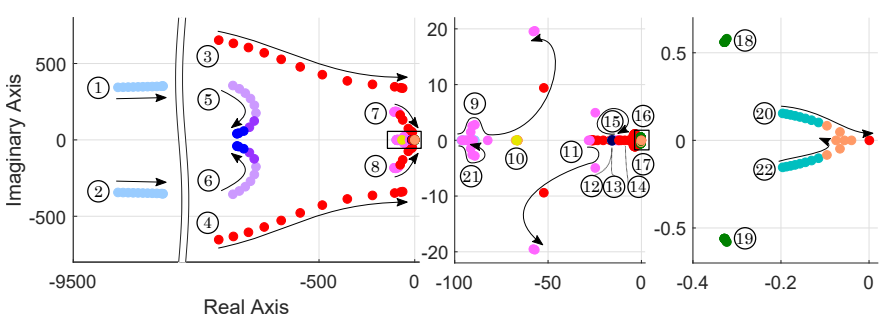

Fig. 14. Pole diagram of Case 2 when $S_{S G}$ is reduced

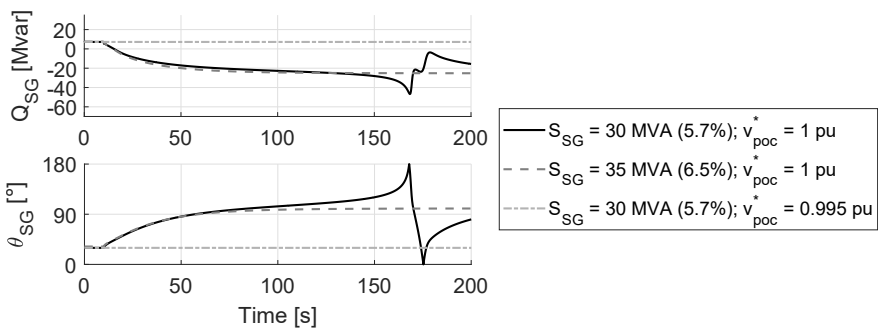

Fig. 15. Time domain results of Case 2

\section{Case 3. Power control and frequency support}

In this case, $S_{S G}$ is reduced from 500 MVA to 200 MVA with variations of $50 \mathrm{MVA}$. Then, below $200 \mathrm{MVA}, S_{S G}$ is reduced until instability with variations of 20 MVA for a better resolution of the results. The value of the f-P droop characteristic $R$ has a great impact in the couplings between the components, as presented in Section III-C. If $R$ is sufficiently high, i.e. the VSC frequency control has a low contribution, the system response tends to Case 1 . Therefore, the minimum synchronous generation required is found for two $R$ values: $5 \%$ and $1 \%$.

When $R$ is set to $5 \%$, the system does not become unstable due to reduction of SG share. It is observed that for a reduction of the SG share down to 1\%, poles 3-4 and 14-18 in Fig. 16a are very low damped, but the system is still stable.

This is validated with the non-linear time domain results in Fig.17. The system, with an SG share of $1 \%$, remains stable after a load step of $1 \%$ at $5 \mathrm{~s}$. The converter compensates the new load and the frequency is kept within proper ranges. It is observed that the dynamics of the frequency are related to the active power of both the SG and the VSC and are 
much faster than in Case 1, where the VSC had no frequency control (see Fig. 13). Then, it is clear that the frequency is coupled to electrical variables instead of the mechanical torque of the machine. This interaction was also observed for a high contribution of the VSC frequency control in Section III-C. Despite of the good response of the frequency, the reactive power injected by the SG to control the voltage exceeds the machine ratings, leading to an unfeasible operational point.

If $R$ is reduced to $1 \%$, interactions between VSC control and SG cause instability. In particular, poles 7 and 8 in Fig. 16b become unstable when $S_{S G}$ is between 115 MVA and 110 MVA (18.7\% and $18.0 \%$ of SG share). These poles correspond to the interaction between PLL and power control of the VSC and the SG identified in Section III-C. The participation of the VSC control is reduced when $S_{S G}$ decreases (poles 7 and 8 become red), but it is still relevant to affect the stability of the mode.

The SG limits are also validated with non-linear time domain simulation in Figs. 18 and 19, where the PLL frequency $f_{p l l}$ and grid frequency $f_{g}$ are compared for $S_{S G}$ equal to 115 MVA and 110 MVA. The system is initialized with a frequency droop characteristic equal to $1.1 \%$, which is reduced to $1 \%$ at $5 \mathrm{~s}$. When $S_{S G}=115 \mathrm{MVA}$, oscillations appear in the frequency measured by the PLL, but the damping of this mode is high enough without affecting the grid frequency, as shown in Fig. 18. However, when $S_{S G}$ is reduced to 110 MVA, the system becomes unstable. The oscillations in the PLL frequency observed in Fig. 19 are caused by the VSC control and are transferred to the rest of the system through the frequency control and active power control of the converter. As a consequence, the system becomes unstable and the grid frequency collapses. The frequency of the oscillations in Figs.18 and 19 are around 68-69 Hz, which agrees with the frequency of the mode associated to poles 7 and 8 .

These two simulations confirm that the origin of the instability is related to the close loop in the VSC control, as discussed in Section III-C. This close loop is highly dependent on the VSC frequency droop gain and becomes unstable when this gain is very high.

\section{Case 4. Power and voltage control and frequency support}

The rated SG power is reduced from 500 MVA until the instability limit with variations of 50 MVA. The minimum synchronous generation required has been found for the same $R$ values as in Case 3. As in Section III-D, this case combines the effects of Case 2 and 3. High $R$ values $(R=5 \%)$ reveal a non-oscillatory angle instability, as in Case 2 . However, the stability limit is reached for higher $S_{S G}$, in particular, between 55 MVA and 50 MVA (9.9\% and $9.1 \%$ of SG share). Low $R$ values lead to instability due to an interaction between the VSC control and the SG as in Case 3. In particular, this stability limit is between 120 MVA and 115 MVA (19.4\% and $18.7 \%$ of SG share).

\section{E. Analytical pole expressions}

In this section, simplified models to derive analytical expressions of the critical system poles are obtained. These models are built including the variables with the highest participation

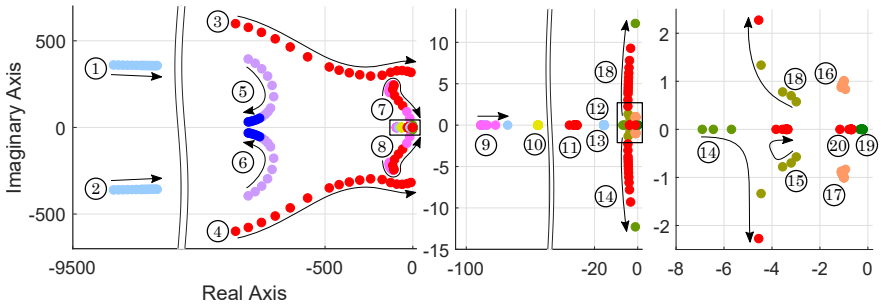

(a)

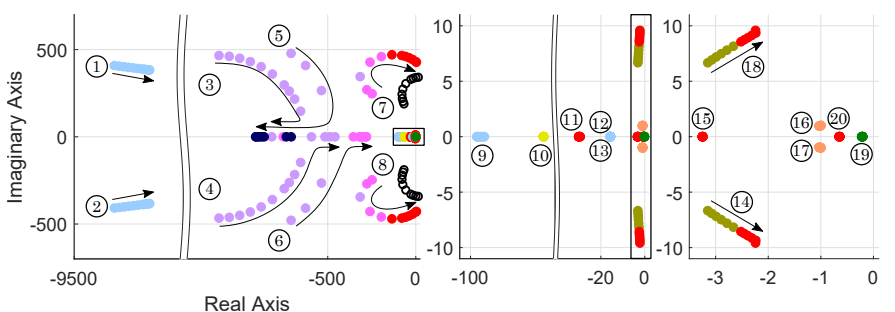

(b)

Fig. 16. Pole diagram of Case 3 when $\mathrm{S}_{\mathrm{SG}}$ is reduced and $R$ is: (a) $5 \%$ and (b) $1 \%$

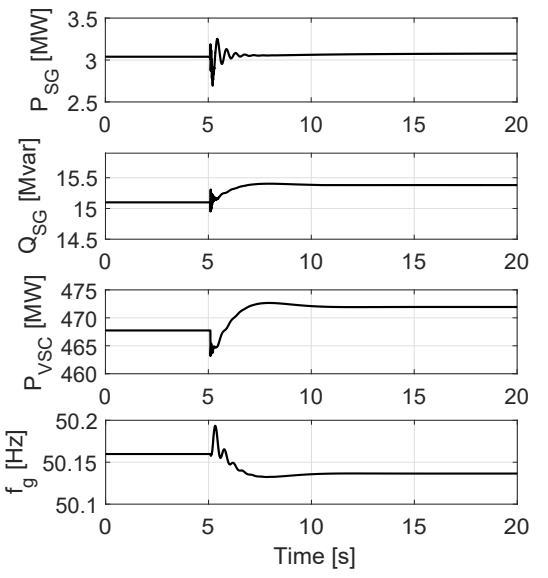

Fig. 17. Time domain results of Case 3 when $R=5 \%$ and $S_{S G}=5 \mathrm{MVA}$
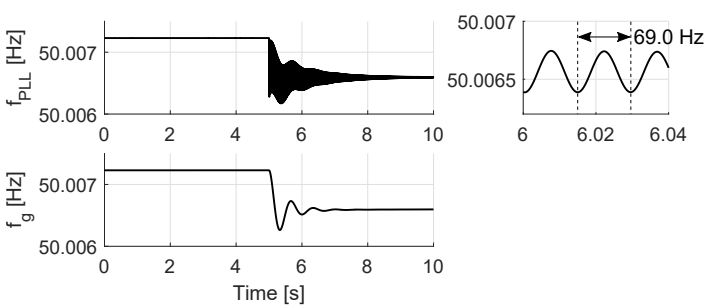

Fig. 18. Time domain results of Case 3 when $R=1 \%$ and $S_{S G}=115 \mathrm{MVA}$
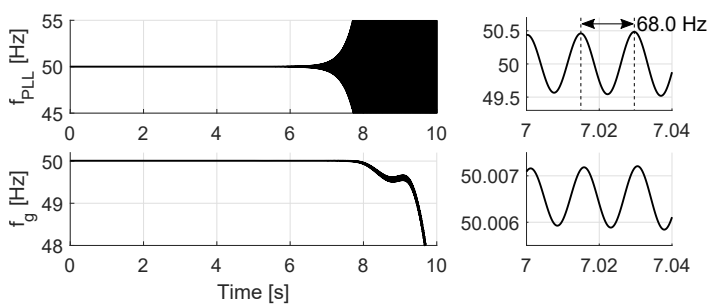

Fig. 19. Time domain results of Case 3 when $R=1 \%$ and $S_{S G}=110 \mathrm{MVA}$ 


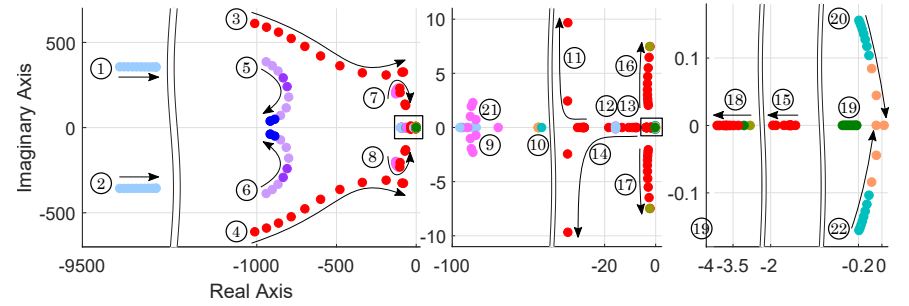

(a)

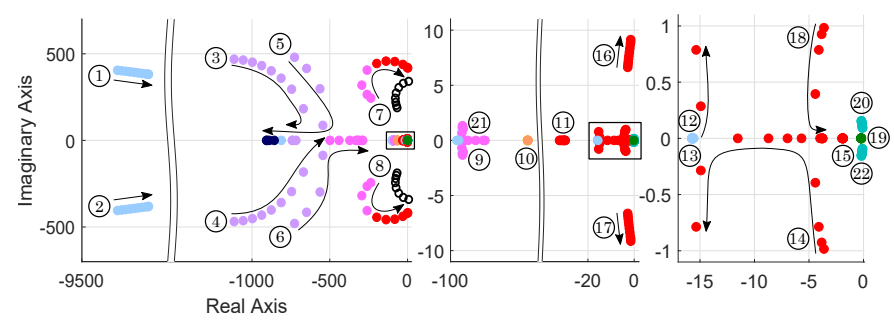

(b)

Fig. 20. Pole diagram of Case 4 when $\mathrm{S}_{\mathrm{SG}}$ is reduced and $R$ is: (a) $5 \%$ and (b) $1 \%$

for each pole, while the rest of the system is removed. Then, analytical pole expressions are obtained from the reduced $\mathbf{A}$ matrices of the simplified models.

This methodology has been applied to find the analytical expressions of the unstable poles found in the previous cases (1-4). Specifically, when frequency support has an important contribution (Cases 3 and 4 , with $R=1 \%$ ), critical system poles can be obtained derived from the simplified A. However, for the other cases, the suggested reduced A matrix is not able to fully capture the dynamics of the rest of the studied poles and a numerical analysis is recommended [32].

For Cases 3 and 4 (with $R=1 \%$ ), the critical poles (7 and 8 ) of the system can be described with a simplified model using four variables: $S e_{p l l}, S e_{P}, i_{s}^{q}$ and $i_{s}^{d}$. Then, the following characteristic equation is derived from the A matrix associated to the simplified model:

$$
\begin{gathered}
\lambda^{4}+\lambda^{3}\left(\alpha_{p a c}+K_{1}\right)+\lambda^{2}\left(K_{1} \alpha_{p a c}+K_{2}\right)+ \\
+\lambda\left(\alpha_{p a c} K_{2}+K_{3} k_{p-p l l}\right)+K_{3} k_{i-p l l} \\
K_{1}=\frac{\left(L_{g}^{q}+L_{g}^{d}\right)\left(R_{g}+R_{d 0}\right)}{L_{g}^{q} L_{g}^{d}} \\
K_{2}=\frac{\left(R_{g}+R_{d 0}\right)^{2}}{L_{g}^{q} L_{g}^{d}}+\omega_{g 0}^{2}, \quad K_{3}=\frac{2 \omega_{g 0} R_{d 0}^{2} K_{f}}{3 V_{p o c 0} L_{g}^{q} \tau_{p a c}}
\end{gathered}
$$

where $\tau_{p a c}$ is the time constant of the VSC power control loop and $L_{g}^{q}$ and $L_{g}^{d}$ are the equivalent SG inductances of the classical model including the effect of subtransient circuits, which are defined as [29]:

$$
\begin{aligned}
& L_{g}^{q}=L_{l}+\left(\frac{1}{L_{m}^{q}}+\frac{1}{L_{k 1}^{q}}+\frac{1}{L_{k 2}^{q}}\right)^{-1} \\
& L_{g}^{d}=L_{l}+\left(\frac{1}{L_{m}^{d}}+\frac{1}{L_{k}^{d}}+\frac{1}{L_{f}^{d}}\right)^{-1}
\end{aligned}
$$

Considering that $\tau_{p a c}$ is in the range of tenths to hundreds of milliseconds, $L_{g}^{q}$ and $L_{g}^{d}$ are equal and in the order of $\mathrm{mH}$, and load resistance $R_{d 0}$ is much larger than the SG internal resistance $R_{g}$, the equation can be simplified as:

$$
\begin{gathered}
\lambda^{4}+\lambda^{3}\left(K_{1}\right)+\lambda^{2}\left(K_{2}\right)+\lambda\left(K_{3} k_{p-p l l}\right)+K_{3} k_{i-p l l} \\
K_{1}=\frac{2 R_{d 0}}{L_{g}^{d}}, \quad K_{2}=\frac{R_{d 0}^{2}}{\left(L_{g}^{d}\right)^{2}}+\omega_{g 0}^{2}, \quad K_{3}=\frac{2 \omega_{g 0} R_{d 0}^{2} K_{f}}{3 V_{p o c 0} L_{g}^{d} \tau_{p a c}}
\end{gathered}
$$

The resulting poles are depicted as black ' $\circ$ ' in Fig. 16b and $20 \mathrm{~b}$ and compared to the poles obtained from the full model. This expression (19) allows to approximately calculate the stability limit when the SG is reduced, within a 5\% of SG share error deviation.

\section{Discussion \& Conclusions}

This paper provides an assessment about the stability limits of a VSC-dominated system considering a VSC with conventional grid-following controls. Two main studies have been conducted: the impact of the VSC control and the minimum synchronous generation to ensure stability.

Grid support controls, i.e. voltage and frequency control, may cause significant interactions between the converter and SG. However, these additional controls can significantly extend the stable operation when SG share is reduced. In particular, this paper draws the following conclusions:

- The system is highly decoupled when voltage or frequency controls are not included in the VSC control.

- VSC voltage control might be coupled with the SG exciter, leading to oscillatory interactions at low frequencies, around $0.01 \mathrm{~Hz}$. These interactions result in a non-oscillatory angle instability when the SG share is reduced below $10 \%$.

- A high contribution of the converter in frequency control causes interactions between the PLL and power control of the VSC and the SG, resulting in oscillations in the range of tens of Hz. Also, the reduction of the SG share may lead to an instability due to this interaction.

- The f-P droop characteristic $R$ is an essential parameter to ensure stability with reduced SG share. In particular, $R$ values around 5\% can ensure stability even for low SG share.

- If the frequency support in the VSC is faster than in the $\mathrm{SG}$, the grid frequency is more coupled with electrical and VSC control variables rather than the mechanical torque of the SG turbine.

It should be noted that the instability due to the voltage control interaction might be affected by the network topology and the system operation. However, the instability related to VSC frequency control can be generalized to other cases since the grid frequency is a global variable of the system.

The stability limits have been determined from a smallsignal analysis, but operational limitations of the VSC and SG have to be contemplated. Also, other studies related to transient analysis, such as faults, must be also considered for a complete assessment of the minimum synchronous generation required. In addition, other VSC control strategies based on grid-forming operation should be compared to the grid support controls used in this paper. 


\section{APPENDIX}

The parameters used in this paper are the following: Synchronous generator: $S_{S G}=500$ MVA, $P_{g r}=70 \% S_{S G}$. Generator: $R_{s}=0.003 \mathrm{pu}, L_{s}=0.15 \mathrm{pu}, L_{d}=1.66 \mathrm{pu}, L_{q}=1.61 \mathrm{pu}, L_{k 1}^{q}=$ $0.7252 \mathrm{pu}, R_{k 1}^{q}=0.00619 \mathrm{pu}, L_{k 2}^{q}=0.125 \mathrm{pu}, R_{k 2}^{q}=0.002368 \mathrm{pu}$, $L_{k}^{d}=0.1713 \mathrm{pu}, R_{k}^{d}=0.0284 \mathrm{pu}, L_{f}^{d}=0.165 \mathrm{pu}, R_{f}^{d}=0.0284 \mathrm{pu}$. Exciter: $K_{A}=200, T_{A}=0.015 \mathrm{~s}, T_{B}=10 \mathrm{~s}, T_{C}=1 \mathrm{~s}$. Turbine: $K_{H P}=0.25, K_{L P}=0.75, T_{R H}=5$ s. Governor: $R_{S G}=5 \%$.

VSC: $S_{V S C}=500 \mathrm{MVA}, R_{c}=0.01 \mathrm{pu}, L_{c}=0.20 \mathrm{pu}$. PLL: $k_{p-p l l}=0.0118, k_{i-p l l}=2.358$. Current control: $\tau_{i}=1 \mathrm{~ms}, \tau_{m}=$ $100 \mu \mathrm{s}$. Power control: $\tau_{P}=10 \mathrm{~ms}, \tau_{p a c}=100 \mathrm{~ms}, k_{p-P}=0.0066$, $k_{i-P}=6.67$. Voltage: $\tau_{V}=10 \mathrm{~ms}, k_{p-V}=0.01, k_{i-V}=10$.

Grid: Voltage: $V_{n}=24 \mathrm{kV}$. Line: $R_{l}=0.03 \Omega / \mathrm{km}$ (at $220 \mathrm{kV}$ ), $L_{l}=1 \mathrm{mH} / \mathrm{km}($ at $220 \mathrm{kV}), l=10 \mathrm{~km}$.

\section{REFERENCES}

[1] ENTSO-E, "High Penetration of Power Electronic Interfaced Power Sources," Tech. Rep. March, 2017.

[2] J. A. Suul, S. D'Arco, P. Rodríguez, and M. Molinas, "Impedancecompensated grid synchronisation for extending the stability range of weak grids with voltage source converters," IET Generation, Transmission \& Distribution, vol. 10, no. 6, pp. 1315-1326, 2016.

[3] J. Z. Zhou, H. Ding, S. Fan, Y. Zhang, and A. M. Gole, "Impact of short-circuit ratio and phase-locked-loop parameters on the small-signal behavior of a VSC-HVDC converter," IEEE Transactions on Power Delivery, vol. 29, no. 5, pp. 2287-2296, 2014.

[4] L. Harnefors, M. Bongiorno, and S. Lundberg, "Input-Admittance Calculation and Shaping for Controlled Voltage-Source Converters," IEEE Transactions on Industrial Electronics, vol. 54, no. 6, pp. 3323-3334, dec 2007.

[5] A. Egea-Alvarez, S. Fekriasl, F. Hassan, and O. Gomis-Bellmunt, "Advanced Vector Control for Voltage Source Converters Connected to Weak Grids," IEEE Transactions on Power Systems, vol. 30, no. 6, pp. 3072-3081, 2015.

[6] L. Xu and L. Fan, "Impedance-based resonance analysis in a vsc-hvdc system," IEEE Transactions on Power Delivery, vol. 28, no. 4, pp. 22092216, 2013.

[7] L. Zhang, L. Harnefors, and H. P. Nee, "Power-synchronization control of grid-connected voltage-source converters," IEEE Transactions on Power Systems, vol. 25, no. 2, pp. 809-820, 2010.

[8] P. Tielens and D. van Hertem, "Grid Inertia and Frequency Control in Power Systems with High Penetration of Renewables," 6th IEEE Young Researchers Symposium in Electrical Power Engineering, no. 2, pp. 1-6, 2012.

[9] M. Zhang, X. Yuan, and J. Hu, "Inertia and Primary Frequency Provisions of PLL-Synchronized VSC HVDC When Attached to Islanded AC System," IEEE Transactions on Power Systems, vol. 33, no. 4, pp. 4179-4188, 2018.

[10] H. P. Beck and R. Hesse, "Virtual synchronous machine," 9th International Conference on Electrical Power Quality and Utilisation, 2007.

[11] K. D. Brabandere, S. Member, B. Bolsens, S. Member, J. V. D. Keybus, S. Member, A. Woyte, and J. Driesen, "A Voltage and Frequency Droop Control Method for Parallel Inverters," IEEE Transactions on Power Electronics, vol. 22, no. 4, pp. 1107-1115, 2007.

[12] S. V. Dhople, B. B. Johnson, and A. O. Hamadeh, "Virtual Oscillator Control for voltage source inverters," 51st Annual Allerton Conference on Communication, Control, and Computing, pp. 1359-1363, 2013.

[13] C. Arghir, T. Jouini, and F. Dörfler, "Grid-forming control for power converters based on matching of synchronous machines," Automatica, vol. 95, pp. 273-282, 2018.

[14] MIGRATE Project, "WP3 - Control and Operation of a Grid with $100 \%$ Converter-Based Devices Deliverable 3.2: Local control and simulation tools for large transmission systems," 2020.

[15] Y. Lin, B. Johnson, V. Gevorgian, V. Purba, and S. Dhople, "Stability assessment of a system comprising a single machine and inverter with scalable ratings," in North American Power Symposium. IEEE, 2017, pp. 1-6.

[16] E. Vittal, M. O'Malley, and A. Keane, "Rotor angle stability with high penetrations of wind generation," IEEE Transactions on Power Systems, vol. 27, no. 1, pp. 353-362, 2012.

[17] S. Laudahn, J. Seidel, B. Engel, T. Bulo, and D. Premm, "Substitution of synchronous generator based instantaneous frequency control utilizing inverter-coupled der," 7th International Symposium on Power Electronics for Distributed Generation Systems, 2016.
[18] G. Pereira, V. Costan, A. Bruyère, and X. Guillaud, "Impact of synchronous machine dynamics on the stability of a power grid with high penetration of variable renewable energies," 15th IET International Conference on AC and DC Power Transmission, 2019.

[19] G. S. Pereira, V. Costan, A. Bruyère, and X. Guillaud, "Synchronous Machine Representations for Stability Studies of Power Systems with Inverters," 13th IEEE PowerTech, 2019.

[20] A. J. Germond and R. Podmore, "Dynamic Aggregation of Generating Unit Models," IEEE Transactions on Power Apparatus and Systems, vol. PAS-97, no. 4, pp. 1060-1069, 1978.

[21] J. Machowski, J. W. Bialek, and J. R. Bumby, Power System Dynamics: Stability and Control. John Wiley \& Sons, 1997.

[22] J. H. Chow, Power System Coherency and Model Reduction. Springer, 2013.

[23] S. Liao, X. Zha, X. Li, M. Huang, J. Sun, J. Pan, and J. M. Guerrero, "A Novel Dynamic Aggregation Modeling Method of Grid-Connected Inverters: Application in Small-Signal Analysis," IEEE Transactions on Sustainable Energy, vol. 10, no. 3, pp. 1554-1564, 2019.

[24] X. Zha, S. Liao, M. Huang, Z. Yang, and J. Sun, "Dynamic Aggregation Modeling of Grid-Connected Inverters Using Hamilton's-Action-Based Coherent Equivalence," IEEE Transactions on Industrial Electronics, vol. 66, no. 8, pp. 6437-6448, 2019.

[25] C. Li, J. Xu, and C. Zhao, "A Coherency-Based Equivalence Method for MMC Inverters Using Virtual Synchronous Generator Control," IEEE Transactions on Power Delivery, vol. 31, no. 3, pp. 1369-1378, 2016.

[26] P. J. Hart, R. H. Lasseter, and T. M. Jahns, "Coherency identification and aggregation in grid-forming droop-controlled inverter networks," IEEE Transactions on Industry Applications, vol. 55, no. 3, pp. 2219-2231, 2019.

[27] P. C. Krause, Analysis of Electric Machinery. McGraw-Hill, 1986.

[28] IEEE Power and Energy Society, IEEE Recommended Practice for Excitation System Models for Power System Stability Studies, 2016.

[29] P. Kundur, Power System Stability and Control. McGrau-Hill, 1994.

[30] L. Harnefors and H.-p. Nee, "Model-Based Current Control of AC Machines Using the Internal Model Control Method," IEEE Transactions on Industry Applications, vol. 34, no. 1, pp. 133-141, 1998.

[31] S.-k. Chung, "A Phase Tracking System for Three Phase Utility Interface Inverters," IEEE Transactions on Power Electronics, vol. 15, no. 3, pp. 431-438, 2000.

[32] G. C. Verghese, F. C. Schweppe, and P. Systems, "Selective Modal Analysis with Applications to Electric Power Systems, Part I: Heuristic Introduction, Part II: The Dynamic Stability Problem," IEEE Transactions on Power Apparatus and Systems, vol. PAS-101, no. 9, pp. 31173134, 1982.

[33] C. Taylor, Power system voltage stability. McGrau-Hill, 1994. 